\title{
Robustness guarantees for linear control designs with an estimated nonlinear model error model
}

\author{
Torkel Glad, Anders Helmersson, Lennart Ljung \\ Division of Automatic Control \\ Department of Electrical Engineering \\ Linköpings universitet, SE-581 83 Linköping, Sweden \\ WWW: http://www. control.isy.liu.se \\ E-mail: torkel@isy.liu.se, andersh@isy.liu.se \\ ljung@isy.liu.se
}

13th February 2004

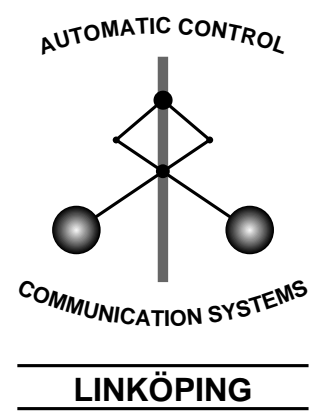

Report no.: LiTH-ISY-R-2592

Submitted to Special issue of IJRNC

Technical reports from the Control \& Communication group in Linköping are available at http://www. control.isy.liu.se/publications. 


\begin{abstract}
Much attention in robust identification and control has been focused on linear low order models approximating high order linear systems. We consider the more realistic situation with a linear model approximating a non-linear system. We describe how a non-linear model error model can be developed, that allows a complete linear design process that results in a closed loop system with performance robustness guarantees (in terms of gain from disturbance to output) against the nonlinear error. Clearly the design can be successful only if the linear model is a reasonably good approximation of the system. A particular aspect of the design process is to define a workable definition of "practical stability" for robust control design, with possible nonlinear model errors. We use affine norms for that purpose.
\end{abstract}

Keywords: identification, control, nonlinear, model error model, robustness 


\title{
Robustness guarantees for linear control designs with an estimated nonlinear model error model
}

\author{
S. T. Glad, A. Helmersson and L. Ljung \\ Department of Electrical Engineering \\ Linköping University \\ SE-581 83 Linköping, Sweden
}

November 29, 2003

\begin{abstract}
Much attention in robust identification and control has been focused on linear low order models approximating high order linear systems. We consider the more realistic situation with a linear model approximating a non-linear system. We describe how a non-linear model error model can be developed, that allows a complete linear design process that results in a closed loop system with performance robustness guarantees (in terms of gain from disturbance to output) against the nonlinear error. Clearly the design can be successful only if the linear model is a reasonably good approximation of the system. A particular aspect of the design process is to define a workable definition of "practical stability" for robust control design, with possible nonlinear model errors. We use affine norms for that purpose.
\end{abstract}

\section{Introduction}

Much attention has been paid to the problem of designing a regulator for a system based on an approximate and uncertain model. There are many useful, classical, results for the case when the model error and uncertainty can be expressed as bounds on the model's Nyquist curve, thus (implicitly) assuming that the true system is linear, e.g. [15]

The common case where the true system may be nonlinear and the design is based on a linear model has also been treated extensively. Among many relevant references we may mention [14], [8], [13].

The problem we consider in this contribution is the following one. A nominal linear model of the system,

$$
y(t)=\hat{G}(q) u(t)
$$

is constructed, e.g. using system identification. (Here $q$ is the shift or derivative operator, depending on the context.) Now such a model cannot exactly describe 
the system. There will be model errors and disturbances that give discrepancies from the model's predictions.

The errors or residuals will be denoted by

$$
\varepsilon(t)=y(t)-\hat{G}(q) u(t)
$$

To be able to say something about the quality of a regulator based on the nominal $\hat{G}$ we must assume something about these residuals. A typical assump-

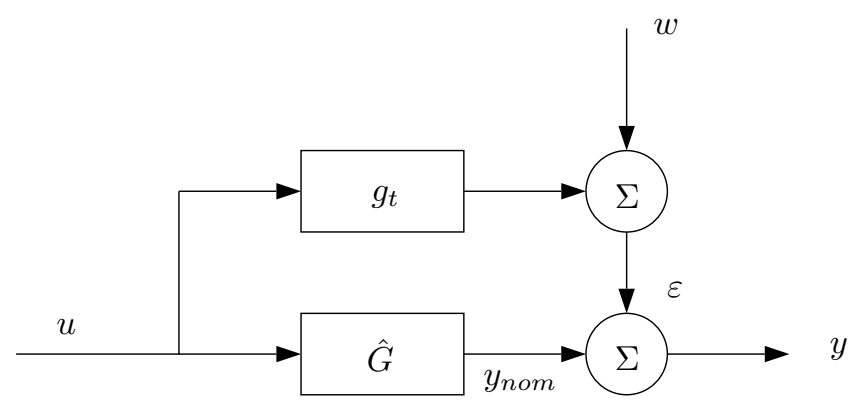

Figure 1: Block diagram of the model error model.

tion, illustrated in Figure 1, is that $\varepsilon$ has one part that originates from the input $u$ ("model error") and one part that is due to disturbances $w(t)$ :

$$
\varepsilon(t)=g_{t}\left(u^{t}\right)+w(t)
$$

Here $u^{t}=\{u(\tau), \tau \leq t\}$. Much work has been devoted to the problem of separating the two effects in (3), e.g. [4], [6], [11], [12],[5],[1]. Often the model error is assumed to be linear (i.e. assuming the true system to be linear):

$$
g_{t}\left(u^{t}\right)=\tilde{G}(q) u(t)
$$

which allows some conventional model validation techniques to be used, e.g. [7], or more sophisticated Toeplitz operator methods, e.g. [12] and [9]. In that case the error model naturally becomes global in the amplitude of $u$.

However, if the true system and hence the error model is possibly nonlinear, the situation is different. We would need error estimates of the kind

$$
\begin{aligned}
\left\|g_{t}\left(u^{t}\right)\right\| & \leq \alpha+\beta\|u\| \\
\|w\| & \leq \gamma
\end{aligned}
$$

(More formal versions of such model error bounds will be discussed in Section 2.) This raises the important question on the validity range of $(5 \mathrm{a})$. On the one hand, these bounds should be global if we intend to apply conventional stability and robustness tools, which are based on variants of the small gain theorem. On the other hand, it will be impossible in practice to validate such bounds for 
unlimited $u$. Moreover, since most real systems are saturating at large input amplitudes, while the nominal model is linear, the relative model error will be 1 at best (at large amplitudes the error will be more or less equal to the output of the nominal model). Such a relative model error is not much to build useful robustness results on.

Now, this seems to be an overly pessimistic view. In practice only inputs of reasonable amplitude will and can be applied to the system, so why bother about unlimited inputs? It seems quite possible to obtain reasonable bounds of the kind

$$
\left\|g_{t}\left(u^{t}\right)\right\| \leq \alpha+\beta\|u\| \text { for }|u(t)| \leq u_{b} \quad \forall t
$$

For a reasonable input bound and a good nominal linear model, it should be possible both to have small $\alpha, \beta$ and to achieve confidence in the bounds by realistic validation tests.

The dilemma is that for many robustness and stability results it is not sufficient to have error bound just in a region of the input space.

The purpose of this paper is to point to some possibilities to deal with this dilemma, that is how to be able to guarantee reasonable behavior of the closed loop system designed from the linear nominal model (1) and knowing error bounds just for bounded inputs as in (6).

\section{Model Error Models and Their Gains}

A model error model is a description of how $u$ affects $\varepsilon$. We will typically not be interested in a detailed such description - that should rather be done as a more detailed nominal model. Our simplest model error model will just require that $\varepsilon$ is bounded provided the input is bounded:

$$
|u(t)| \leq u_{b}, \quad t \leq \tau \Rightarrow|\varepsilon(\tau)| \leq \varepsilon_{\max }
$$

We will also look at bounds on the gain of such a model. To go well together with linear control design, we shall work with combinations of frequency weightings and unstructured, unknown models with bounded (estimated) gain. That means that the general structure of the model from $u$ to $\varepsilon$ can be depicted as in Figure 2. Formally we have

$$
\begin{aligned}
\varepsilon(t) & =\varepsilon_{F}(t)+w(t)=W_{2}(q) z_{2}(t)+w(t) \\
z_{2}(t) & =\tilde{g}_{\text {mem }}\left(z_{1}^{t}\right), \quad z_{1}(t)=W_{1}(q) u(t)
\end{aligned}
$$

Here, $W_{1}$ and $W_{2}$ are given linear filters and $\tilde{g}_{m e m}$ is an uncertain model. The bottom line is that, with some amount of confidence, based on the measured data, and possibly on prior information, we should be able to say that its gain is bounded, provided the input is bounded. The model $\tilde{g}_{m e m}$ can come in different shapes. It could be

- Linear Time Invariant. In this case we can take either $W_{1}$ or $W_{2}$ to be unity, since all the blocks in in Figure 2 commute. 


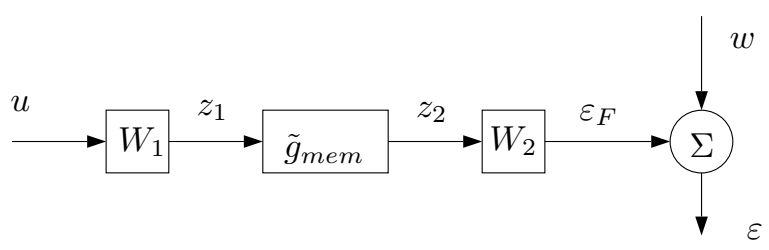

Figure 2: The model error model

- Linear Time Varying.

- General Non-Linear.

\section{The nonlinear gain definition}

An important aspect of nonlinear model error models is that the standard definition of gain for a nonlinear system with input $u$ and output $y$ :

$$
\sup _{u \neq 0} \frac{\|y\|}{\|u\|}
$$

is sometimes misleading. Consider for instance a static nonlinearity of the form shown in Figure 3. The gain becomes $+\infty$ no matter how small $\epsilon$ is. This

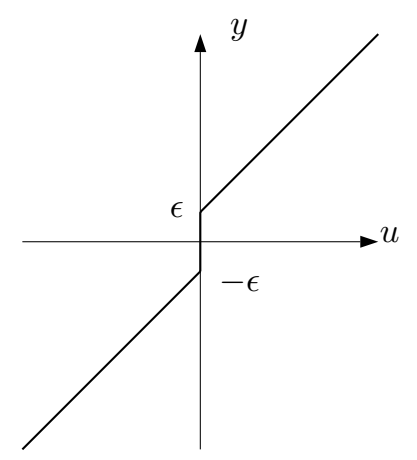

Figure 3: Static nonlinearity with discontinuity at the origin

type of nonlinearity is quite common in actuators and power amplifiers where positive and negative signals are handled by different physical components, that are not perfectly matched.

We propose instead to use an affine version of the gain definition. This has been used in the nonlinear control literature, see e.g. Vidyasagar [13]. We use 
a power gain definition similar to $[3,2]$ and say that the nonlinear system has gain $\beta$ with offset $\alpha$ if there are positive constants $\alpha$ and $\beta$ such that

$$
|u(t)| \leq u_{b}, \quad t \leq T \Rightarrow\left\|z_{2}\right\|_{T} \leq \alpha \sqrt{T}+\beta\left\|z_{1}\right\|_{T}
$$

for all input-output pairs $z_{1}$ and $z_{2}$, consistent with $|u(t)| \leq u_{b}$ and all positive $T$. Here $\|\cdot\|_{T}$ denotes the following truncated norm:

$$
\|z\|_{T}^{2}=\int_{0}^{T} z(t)^{2} d t
$$

For the nonlinearity in Figure 3 we could for instance take $\alpha=\epsilon$ and $\beta=1$.

\section{$3 \quad$ Linear Control Design}

The presence of an amplitude constraint in the model error model means that the controller design also has to take this constraint into account. There are two ways of doing this. Either one can make a linear design and look for conditions that guarantee that the resulting control signal amplitude does not exceed the bound $u_{b}$. Or else one can design a nonlinear controller that forces the control to always satisfy the bound $|u| \leq u_{b}$. In this section we look at linear designs.

If a linear regulator $-F$ is used, the closed loop system is given by the block diagram of Figure 4, where we have also introduced a model for the disturbance $w$ as an external signal $e$ passing through a linear system $G_{d}$. From the block

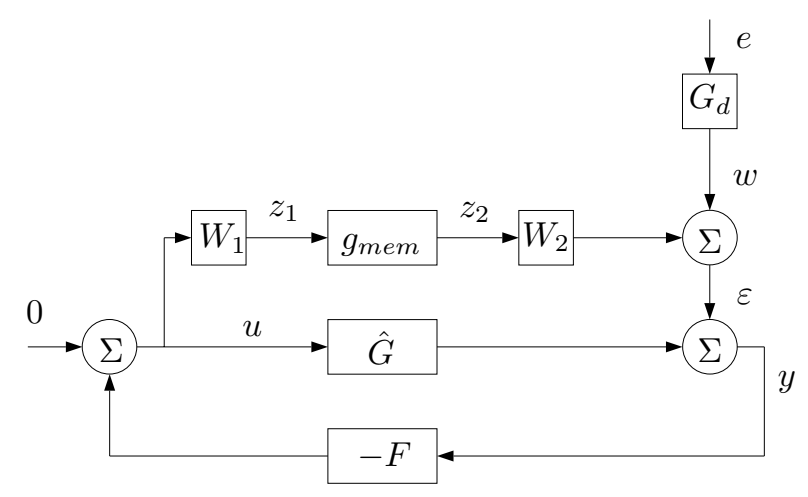

Figure 4: Block diagram of feedback loop with model error and linear regulator.

diagram it follows that the control signal can be written

$$
u=-(I+F \hat{G})^{-1} F \varepsilon
$$

Let $h$ denote the impulse response corresponding to the transfer function $(I+$ $F \hat{G})^{-1} F$. Since this transfer function might contain a direct term, we write $h$ 
in the form

$$
h(t)=h_{d} \delta(t)+h_{c}(t)
$$

where $h_{c}$ is an ordinary function. We will use the norm

$$
\|h\|_{1}=\left|h_{d}\right|+\int_{0}^{\infty}\left|h_{c}(t)\right| d t
$$

where we have assumed that the closed loop system has all its poles in the left half plane so that the integral exists.

Then we have the following

Proposition 1 Let the linear systems $F$ and $\hat{G}$ be initialized with the zero state at $t=0$. Assume that the model error model (7) is satisfied, i.e.

$$
|u(t)| \leq u_{b}, \quad t \leq \tau \Rightarrow|\varepsilon(\tau)| \leq \varepsilon_{\max }
$$

A sufficient condition for

$$
|u(t)|<u_{b}, \quad t \geq 0
$$

to be satisfied is that

$$
\|h\|_{1}<\frac{u_{b}}{\varepsilon_{\max }}
$$

Proof. Since the linear systems are initialized with zero,

$$
|u(0)|=\left|h_{d}\right| \varepsilon(0) \leq\|h\|_{1} \varepsilon_{\max }<u_{b}
$$

Now assume that $|u(t)| \geq u_{b}$ for some $t>0$. Since $u$ is continuous it follows that there exists a $t_{1}>0$ such that $|u(t)|<u_{b}$ for $0 \leq t<t_{1}$ and $\left|u\left(t_{1}\right)\right|=u_{b}$. The model error model is then valid on the interval $0 \leq t \leq t_{1}$ and $\varepsilon(t) \leq \varepsilon_{\max }$ on that interval. It follows that, for $0 \leq t \leq t_{1}$, we have

$$
|u(t)| \leq\left(\left|h_{d}\right||\varepsilon(t)|+\int_{0}^{t}|h(\tau)||\varepsilon(t-\tau)| d \tau \leq \varepsilon_{\max }\|h\|_{1}<u_{b}\right.
$$

Setting $t=t_{1}$ we get a contradiction, which shows that $|u(t)|<u_{b}$ for all positive $t$.

Remark If we do not have any information on $\varepsilon$ except the bound $\varepsilon_{\max }$, then the sufficient condition (16) becomes necessary by replacing $<$ with $\leq$. This can be seen from (17), where it is possible to obtain equality by using an $\varepsilon$ having the same sign as $h$. 


\section{Control properties}

Consider again the system of Figure 4. We will now consider the ability of the controller to suppress the disturbance $w$ in the presence of the model error. To do that we assume that the model error model satisfies both (7) and (11), i.e. we have a bound on the amplitude of $\varepsilon$ but also a bound on the gain of $g_{m e m}$. The external disturbance $w$ is modeled as a signal $e$ passing through the linear system $G_{d}$. The transfer function $G_{d}$ can thus be used to model the frequency content of $w$.

Let $S$ and $T$ be the nominal sensitivity and complementary sensitivity functions of the closed loop system (without the error model):

$$
S=\frac{1}{1+\hat{G} F}, \quad T=\frac{\hat{G} F}{1+\hat{G} F}
$$

Also define the transfer functions

$$
G_{z}=T \frac{W_{1} W_{2}}{\hat{G}}=S F W_{1} W_{2}, \quad G_{w}=W_{1} S F
$$

We now have the following property of the closed loop system.

Theorem 1 Let the system be described by Figure 4 and assume that the model error model (7), (11), is satisfied, i.e.

$$
\begin{aligned}
& |u(t)| \leq u_{b}, \quad t \leq \tau \Rightarrow|\varepsilon(\tau)| \leq \varepsilon_{\max } \\
& |u(t)| \leq u_{b}, \quad t \leq T \Rightarrow\left\|z_{2}\right\|_{T} \leq \alpha \sqrt{T}+\beta\left\|z_{1}\right\|_{T}
\end{aligned}
$$

Also assume that the transfer functions $S, T, W_{1}$ and $W_{2}$ have their poles strictly in the left half plane, that (16) of Proposition 1 is satisfied, i.e. $\|h\|_{1}<u_{b} / \varepsilon_{\max }$, that $\beta\left\|G_{z}\right\|<1$ and that the linear systems are initialized with zero initial state. Then the output $y$ is bounded by

$$
\|y\|_{T} \leq\left\|S W_{2}\right\| \frac{\alpha \sqrt{T}}{1-\beta\left\|G_{z}\right\|}+\left(\frac{\beta\left\|S W_{2}\right\|\left\|G_{w} G_{d}\right\|}{1-\beta\left\|G_{z}\right\|}+\left\|S G_{d}\right\|\right)\|e\|_{T}
$$

Proof. Since the properties of Proposition 1 are satisfied it follows that $|u| \leq u_{b}$ so that the model error model can be used. Following the signals around the block diagram of Figure 4 gives the relations

$$
\begin{aligned}
z_{1} & =-G_{z} z_{2}-G_{w} G_{d} e \\
y & =S W_{2} z_{2}+S G_{d} e
\end{aligned}
$$

The relation (11) together with (19) then gives

$$
\left\|z_{2}\right\|_{T} \leq \alpha \sqrt{T}+\beta\left\|z_{1}\right\|_{T} \leq \alpha \sqrt{T}+\beta\left\|G_{z}\left|\|\| z_{2}\left\|_{T}+\right\| G_{w} G_{d}\|\|\right|\right\|_{T}
$$

Solving for $z_{2}$ then gives

$$
\left\|z_{2}\right\|_{T} \leq \frac{\alpha \sqrt{T}+\beta\left\|G_{w} G_{d}\right\|\|e\|_{T}}{1-\beta\left\|G_{z}\right\|}
$$


From (20) we get

$$
\|y\|_{T} \leq\left\|S W_{2}\right\|\left\|z_{2}\right\|_{T}+\left\|S G_{d}\right\|\|e\|_{T}
$$

and substituting (21) gives (18).

\section{Controllers that Limit the Control Amplitude}

A disadvantage with linear controllers in our context is that a large unexpected disturbance might cause the controller to output a control with $|u|>u_{b}$. The model error model then gives no information on $\varepsilon_{\max }$ and it is not possible to say anything about the control system behavior. To overcome this difficulty it is natural to look at controllers that limit the control amplitude so that $|u(t)| \leq u_{b}$ is always satisfied. There are several different methods for the design of controllers that have a limited output. They include anti-windup techniques, Model Predictive Control (MPC) and various optimal control schemes. Here we will not propose a particular scheme, but discuss a controller with a certain structure. Let the model $\hat{G}$ have a state space realization

$$
\dot{x}=A x+B u, \quad y_{n o m}=C x, \quad \text { i.e. } y=C x+\varepsilon
$$

where $A$ has all its eigenvalues strictly in the left half plane. We assume that the control is generated by a nonlinear controller

$$
u=-k(x), \quad|k(x)| \leq u_{b}, \quad k(x)=L x \text { in nbh of } 0
$$

with an accompanying Lyapunov function $V(x)$. We will assume that $V$ is positive definite, radially unbounded, $V(0)=0$ and that

$$
V_{x}(A x-B k(x)) \leq-x^{T} Q x
$$

for some positive definite matrix $Q$. Also we assume a bound on the gradient of $V$ :

$$
\left|V_{x}(x)\right| \leq c_{V}|x|
$$

Since the control is assumed to be linear in some neighborhood of the origin, there is some level set of $V$ such that

$$
V(x) \leq d_{\ell} \Rightarrow k(x)=L x
$$

for some positive $d_{\ell}$. Two cases where we get controllers of the form we propose is in MPC with quadratic cost and LQ with saturations. Since a quadratic criterion is minimized, the optimum is a linear feedback as long as no constraints are active.

We assume that the state feedback is combined with a linear observer in the following manner.

$$
\begin{aligned}
& u=-k(\hat{x}) \\
& \dot{\hat{x}}=A \hat{x}+B u+K(y-C \hat{x}) \\
& y=y_{n o m}+\varepsilon=C x+\varepsilon
\end{aligned}
$$


Introducing the observer error $\tilde{x}=x-\hat{x}$, gives the observer dynamics

$$
\dot{\tilde{x}}=(A-K C) \tilde{x}-K \varepsilon
$$

It follows that

$$
\tilde{x}(t)=e^{(A-K C) t} \tilde{x}(0)+\int_{0}^{t} e^{(A-K C) \tau} K \varepsilon(t-\tau) d \tau
$$

Defining

$$
c_{O}(K)=\int_{0}^{\infty}\left|e^{(A-K C) \tau} K\right| d \tau
$$

we see that the observer error magnitude $\tilde{x}$ will eventually decrease to $c_{O}(K) \varepsilon_{\max }$, if the model error model (7) is used.

The following result allows us to use linear analysis techniques for the system.

Theorem 2 Suppose a system is modeled by (22) and controlled with a controllerobserver satisfying (23), (24), (25), (26) and (27). Assume that the model error satisfies (7), i.e.

$$
|u(t)| \leq u_{b}, t \leq \tau \Rightarrow|\varepsilon(\tau)| \leq \varepsilon_{\max }
$$

Consider the level set

$$
V(\hat{x}) \leq d
$$

for some $d>0$. Then there exists a set $\mathcal{O}$ of observer gains, such that

(i) for every $K \in \mathcal{O}$ there exists a $T(K)$ such that $\hat{x}(t)$ lies in (30) for all $t \geq T(K)$.

(ii) the set $\mathcal{O}$ contains a neighborhood of the origin. (In particular there are nonzero gains in $\mathcal{O}$.)

Proof. From (28) it follows that there exists a $K_{o}$ and $T_{o}$ such that for $|K| \leq K_{o}$ and $t>T_{o}$

$$
|\tilde{x}(t)| \leq \underbrace{2 \int_{0}^{\infty}\left|e^{A \tau}\right| d \tau}_{c_{A}}|K| \varepsilon_{\max }
$$

Now consider $V(\hat{x})$.

$$
\begin{aligned}
& \frac{d}{d t} V(\hat{x})=V_{x}(\hat{x})(A \hat{x}-B k(\hat{x})+K(C \tilde{x}+\varepsilon)) \leq \\
&-\hat{x}^{T} Q \hat{x}+c_{V}|\hat{x}||K|\left(|C||\tilde{x}|+\varepsilon_{\max }\right) \leq \\
&-|\hat{x}|\left(\lambda_{Q \min }|\hat{x}|-|K| c_{V} \varepsilon_{\max }\left(|C| c_{A}|K|+1\right)\right)
\end{aligned}
$$

where $\lambda_{Q \min }$ is the smallest eigenvalue of $Q$. It follows that $\dot{V}<0$ for $|\hat{x}|>$ $c_{o}=|K| c_{V} \varepsilon_{\max }\left(|C| c_{A}|K|+1\right) / \lambda_{Q \min }$. Now let $c_{1}=\inf \left\{|x|: V(x)>d_{\ell}\right\}$ and 
choose $K$ such that $c_{o}<c_{1}$. It follows that $\dot{V}<0$ for $\hat{x}$ outside the set given by (30). Consequently $\hat{x}$ will eventually reach that set.

Remark 1. The theorem can in particular be applied to the case $d_{\ell}=d$. It then guarantees that $\hat{x}$ will eventually converge to the set (26) where the control is linear.

Remark 2. It follows from the proof of the theorem that it is possible to make the observer error $\tilde{x}$ as small as one wishes asymptotically. This is done by making the observer gain small and is thus at the expense of the observer settling time. Note also that a small $\tilde{x}$ only means that $\hat{x}$ is close to $x$ of the model. There is still the model error $\varepsilon$ to take into account.

Remark 3. The theorem gives conditions for $\hat{x}$ to converge to a certain region. Since we have expressions for the observer error in (28) and (29) this also implies that $x$ will converge to some level set of $V$ that we can compute.

If the Lyapunov function $V$ is actually quadratic the estimates can be made more precise.

Theorem 3 Let the conditions of Theorem 2 be satisfied and assume that $V$ is quadratic, $V(x)=x^{T} P x$ for some positive definite symmetric matrix $P$. Let $\lambda$ be the largest eigenvalue of $P$ and let $\mu$ be the smallest eigenvalue of $P^{-1} Q$. Then $\hat{x}$ will eventually enter and remain in the level set

$$
\hat{x}^{T} P \hat{x} \leq r^{2}
$$

provided $r$ satisfies

$$
r>\frac{2 \sqrt{\lambda}}{\mu}|K|\left(|C| c_{O}(K)+1\right) \varepsilon_{\max }
$$

Proof. Differentiating the Lyapunov function gives

$$
\dot{V} \leq-\hat{x}^{T} Q \hat{x}+2 \hat{x}^{T} P|K|\left(|C||\tilde{x}|+\varepsilon_{\max }\right)
$$

Evaluating the right hand side on a level set $V=r^{2}$ gives

$$
\dot{V} \leq-\mu r^{2}+2 \sqrt{\lambda} r|K|\left(|C||\tilde{x}|+\varepsilon_{\max }\right)
$$

From this expression and (28), (29) the statement of the theorem follows.

From these theorems we can derive a result on disturbance rejection.

Theorem 4 Assume that the conditions of Theorems 1 and 2 are satisfied and that $d \leq d_{\ell}$. Then there exists some $t_{0}$ such that for $t>t_{0}$ the controller is described by the transfer function

$$
F(s)=L(s I-A+B L+K C)^{-1} K
$$

and the estimates (18) apply (Here the norm of (12) has to be interpreted with the origin of the time axis shifted to $\left.t_{0}\right)$. 
Proof. It follows from Theorem 2 that $\hat{x}$ will enter and remain in the set $V \leq d \leq d_{\ell}$, where the control is linear. The transfer function $F$ is then given by the combination of linear feedback and observer dynamics. Since the system operates as a linear system the calculations of Theorem 1 can be carried through. Those calculations assume a zero initial state, but the incorporation of a nonzero initial state only gives the superposition of an exponentially decaying additive term whose influence can be neglected if $t_{0}$ is large enough.

\section{Example}

Consider the nonlinear system described in Figure 5. Suppose $\hat{G}$ is the lineariza-

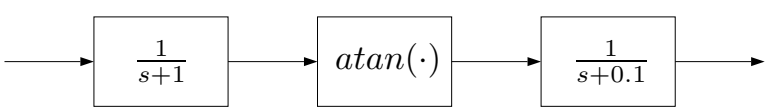

Figure 5: Physical system used in example.

tion of this system around the origin:

$$
\hat{G}=\frac{1}{(s+1)(s+0.1)}
$$

Then we could use the the model error model of Figure 2 where

$$
W_{1}=\frac{1}{s+1}, \quad W_{2}=\frac{1}{s+0.1}
$$

and $g_{m e m}$ satisfies (7), (11) with

$$
u_{b}=1, \quad \varepsilon_{\max }=1, \quad \alpha=0, \quad \beta=0.2
$$

Since $\varepsilon_{\max }=1, u_{b}=1$ and the gain of the model error model is 0.2 the maximum amplitude of the external signal $w$ is 0.8 . Using the state space representation

$$
\dot{x}=\left[\begin{array}{cc}
-1.1 & -0.1 \\
1 & 0
\end{array}\right] x+\left[\begin{array}{l}
1 \\
0
\end{array}\right] u, \quad y=\left[\begin{array}{ll}
0 & 1
\end{array}\right] x
$$

a controller of the form (27) can be made using the following data.

$$
P=\left[\begin{array}{ll}
5 & 5 \\
5 & 6
\end{array}\right], \quad Q=I, \quad L=B P=\left[\begin{array}{ll}
5 & 5
\end{array}\right], \quad K=\left[\begin{array}{l}
0.14 \\
1.13
\end{array}\right]
$$

Since $\|h\|_{1}=0.975$ (see (15)) it follows that the controller will operate in the linear region if it is initialized with zero initial state. Evaluating (18) for $G_{d}=0.01 /(s+0.01)$ (i.e. we model $w$ as a very slowly varying signal) we have $\alpha=0$ and

$$
\|y\|_{T} \leq 0.19\|e\|_{T}
$$


If there had been no model error we would have had

$$
\|y\|_{T} \leq\left\|S G _ { d } \left|\|||\|_{T}=0.16\|\mid\|_{T}\right.\right.
$$

We conclude that in this particular case the model error only degrades the performance marginally.

\section{Discussion}

Of course it is impossible to give absolute robustness guarantees for a design based only on identification data (and hence on a finite number of limited precision measurements) and with no a priori knowledge of the physical system. The basic problem is that estimates of the size of the error can only be validated in restricted parts of the input domain. The problem of the control design is thus to establish that the controller guarantees that the delivered input stays in this restricted region at the same time as stability is ensured.

There are many techniques and results that may guarantee the stability of the closed loop system obtained by a linear regulator and a non-linear controller. Most of them, however require global descriptions of the model error. In this paper we have tried to establish results that do not rely upon global error descriptions.

Theorem 1 shows that a precise robustness result can be proven if the parameters $\alpha$ and $\beta$ of the affine gain are known, even if these gains are restricted to a certain region of the input space. This shows that one aim of the identification procedure could be to give as reliable estimates as possible for those parameters. Note that the result of the theorem does not give asymptotic stability in the case of $\alpha>0$. Instead an upper bound on $\|y\|$ is given. This is natural, since $g$ might contain a nonlinearity of e.g. the form shown in Figure 3. Then we could expect a limit cycle in the closed loop system. However, the result of Theorem 1 gives an upper bound on the amplitude in that case.

A restriction with Theorem 1 is that it assumes that the regulator starts under well-defined conditions. It does not address if the system may be stabilized starting with big control errors, and/or large disturbances. Theorems 2 and 3 show that also this situation can be handled with properly chosen regulators that limit the control input but behave linearly for smaller control errors.

There are several ways in which the gain of $g$ could be estimated. One way would be to estimate the parameters of a fairly general nonlinear black box structure, e.g. neural nets, piecewise linear models or local models. From the identified model, bounds on the gain could be calculated. For a discussion of nonlinear black box models, see [10]. An important topic is of course how the identification procedure is affected by the fact that we are only interested in the gain and not the details of the model.

\section{Acknowledgment}

This work has been supported by the Swedish Research Council. 


\section{References}

[1] X. Bombois, M. Gevers, and G Scorletti. A measure of robust stability for an identified set of parameterized transfer functions. IEEE Trans. Autom. Control, AC-45(11):2124-2145, Nov 2000.

[2] Peter M. Dower. Comments on $l_{2}$-gain analysis of systems with persistent outputs. In Proceedings of the 39th CDC, pages 263-268, 2000.

[3] Peter M. Dower and Matthew R. James. Stability of nonlinear systems with worst case power gain disturbances. In Proceedings of the 37th $C D C$, pages 4075-4080, 1998.

[4] G.C. Goodwin, M. Gevers, and B. Ninness. Quantifying the error in estimated transfer functions with application to model order selection. IEEE Trans. Automatic Control, 37(7):913-929, 1992.

[5] R.G. Hakvoort and P.M. van den Hof. Identification of probabilistic system uncretianty regions by explicit evaluation of bias and variance errors. IEEE Trans. Autom. Control, AC-42(11):1516-1528, Nov 1997.

[6] L. Ljung. Model validation and model error models. In B.Wittenmark and A. Rantzer, editors, The Aström Symposium on Control, pages 15-42. Studentlitteratur, Lund, Sweden, August 1999.

[7] Lennart Ljung and Lei Guo. The role of model validation for assessing the size of the unmodeled dynamics. IEEE Trans.Automatic Control, AC42(9):1230-1240, Aug 1997.

[8] A. Megretski and A. Rantzer. System analysis via integral quadratic constraints. IEEE Trans. Autom. Control, AC-42(6):819-830, June 1997.

[9] K. Poolla, P. Khargonekar, A. Tikku, J. Krause, and K. Nagpal. A time domain approach to model validation. IEEE Trans. Automatic Control, AC-39:951-959, 1994.

[10] J. Sjöberg, Q. Zhang, L. Ljung, A. Benveniste, B. Delyon, P.Y. Glorennec, H. Hjalmarsson, and A. Juditsky. Nonlinear black-box modeling in system identification: A unified overview. Automatica, 31(12):1691-1724, 1995.

[11] R. Smith and J. C. Doyle. Model validation: a connection between robust control and identification. IEEE Trans. Automatic Control, AC-37:942952, 1992.

[12] R. Smith and G.E. Dullerud. Continuous-time control model validation using finite experimental data. IEEE Trans. Automatic Control, AC-41:10941105, 1996.

[13] M. Vidyasagar. Nonlinear Systems Analysis. Prentice-Hall, Englewood Cliffs, NJ, 1993. 
[14] V.A. Yakubovich. The method of matrix inequalities in the theory of stability of nonlinear systems. Avtomatika i Telemekhanika, 25(7):1017-1029, 1964.

[15] Kemin Zhou, John C. Doyle, and Keith Glover. Robust and Optimal Control. Prentice Hall, 1996. 